\title{
Holographic entanglement and causal shadow in time-dependent Janus black hole
}

\author{
Yūki Nakaguchi, ${ }^{a, b}$ Noriaki Ogawa $^{c}$ and Tomonori Ugajin ${ }^{d}$ \\ ${ }^{a}$ Institute for the Physics and Mathematics of the Universe, University of Tokyo, \\ Kashiwa, Chiba 277-8583, Japan \\ ${ }^{b}$ Department of Physics, Faculty of Science, University of Tokyo, \\ Bunkyo-ku, Tokyo 133-0022, Japan \\ ${ }^{c}$ Quantum Hadron Physics Laboratory 8 Mathematical Physics Laboratory, \\ RIKEN Nishina Center, Wako, Saitama 351-0198, Japan \\ ${ }^{d}$ Kavli Institute for Theoretical Physics, University of California, \\ Santa Barbara, CA 93106, U.S.A. \\ E-mail: yuuki.nakaguchi@ipmu.jp, noriaki@riken.jp, \\ ugajin@kitp.ucsb.edu
}

ABSTRACT: We holographically compute an inter-boundary entanglement entropy in a time-dependent two-sided black hole which was constructed in [1] by applying timedependent Janus deformation to BTZ black hole. The black hole contains "causal shadow region" which is causally disconnected from both the conformal boundaries. We find that the Janus deformation results in an earlier phase transition between the extremal surfaces and that the phase transition disappears when the causal shadow is sufficiently large.

KEYwords: Black Holes in String Theory, AdS-CFT Correspondence

ARXiv EPRINT: 1412.8600 


\section{Contents}

1 Introduction 1

2 Properties of three-dimensional Janus Black Hole 3

2.1 The three-dimensional Janus metric 3

2.1.1 Time-dependent Janus deformation of BTZ metric 3

2.1.2 Dual CFT coordinate $(t, \theta)$ and UV cutoff $\epsilon_{\mathrm{CFT}} \quad 3$

2.1.3 As a solution of Einstein-scalar theory 4

2.2 Main differences from BTZ black hole 5

2.2.1 Causal shadow region 5

2.2.2 Time-dependence 5

2.3 The CFT interpretation of the Janus black hole 5

3 Calculation of holographic entanglement entropy $\quad 6$

$\begin{array}{ll}3.1 \text { Covariant holographic entanglement entropy } & 6\end{array}$

3.2 Extremal areas in connected phase 8

3.3 How to calculate extremal areas in disconnected phase 8

$\begin{array}{lll}\text { 3.3.1 Solving the equation of motion } & 9\end{array}$

$\begin{array}{ll}\text { 3.3.2 Returning point }\left(y_{*}, t_{*}\right) & 9\end{array}$

$\begin{array}{lll}\text { 3.3.3 Extremal surface area } & 10\end{array}$

3.4 Some limits of extremal surface areas in disconnected phase 11

3.4.1 Early time limit for large subsystem $\left(\theta_{\infty} \gg t_{\infty}\right) \quad 11$

3.4.2 Late time limit for large subsystem $\left(t_{\infty} \gg \theta_{\infty} \gg r_{0}^{-1}\right) \quad 12$

3.4.3 Up to the lowest order of $\gamma^{2} \quad 12$

4 Time evolution of entanglement entropy and phase transition $\quad 13$

$\begin{array}{llr}5 & \text { Conclusions } & 16\end{array}$

$\begin{array}{ll}\text { A The } \gamma \text {-expansion of holographic entanglement entropy } & 17\end{array}$

\section{Introduction}

The relation between entanglement and black hole interior has attracted much attention recently [2-5]. For eternal AdS black holes, it was discussed that the time evolution of holographic entanglement entropy [6-8] can capture some information about the black hole interior, taking a particular time slicing with which the black hole looks time dependent. For subsystems composed of two disjoint same intervals located in each of two CFT's, the original CFT and the thermofield doubled copy CFT [9], its holographic entanglement 
entropy grows linearly in time for a while, in accordance with the growth of the wormhole inside the black hole. At a certain critical time, the entropy becomes saturated at twice the value of the black hole thermal entropy. In the dual CFT language, this time dependent behavior of the entanglement entropy is interpreted in terms of global quench process [10]. For such Calabrese Cardy type of two dimensional quenches, a systematic construction of their holographic duals was discussed in [11].

More general two-sided black holes can have even richer interior structures. For example, similar inter-boundary entanglement entropies in charged or rotating black hole geometries, which have vertically extended Penrose diagrams, were investigated in $[12,13]$. In this paper, we focus on another interesting class of two-sided black holes with a so called "causal shadow" region, which is a bulk region causally inaccessible from both the boundaries. The implications of such a region for holographic entanglement entropy have been discussed [14, 15]. For example, we can construct an asymptotically AdS black holes with a causal shadow by sending shock waves from the boundaries of eternal AdS black holes [16-18], and we can also discuss its dual CFT [19]. It is an interesting question how the dual CFT encodes information on causal shadow regions.

To investigate this question further, we concentrate on another type of black hole with a causal shadow called the three-dimensional time-dependent Janus black hole, ${ }^{1}$ which is a one parameter deformation of the BTZ black hole and a solution of the Einstein-scalar theory [1]. This black hole geometry has a nontrivial dilaton configuration, without which it reduces to just the eternal BTZ black hole. From the viewpoint of the dual boundary theory, this nontrivial dilaton configuration corresponds to the difference in the coupling constant and so in Hamiltonian between the two CFT's, the original CFT and the thermofield doubled copy CFT [1]. Its corresponding CFT state was proposed [21] as a natural extension of the usual eternal AdS black hole/thermofield double state correspondence [22, 23], and this proposal was checked by computing a one point function both on the CFT side and the gravity side $[1,21]$.

In this paper, we study the time evolution of an inter-boundary holographic entanglement entropy in the Janus black hole geometry, expecting to capture some information on its causal shadow. As in the BTZ black hole geometry, there are two extremal surfaces for the subsystem we take, where the entanglement entropy is given as the area of the one with the smaller area. One which we call "connected surface" passes through the black hole interior and connects the two asymptotic boundaries, while the other which we call "disconnected surface" does not pass through the black hole interior but can penetrate partially into the interior.

In the Janus black hole geometry with not so large deformation parameter, there is a critical time $t_{c}$ at which the surface giving the entanglement entropy switches from the connected one to the disconnected one, as in the BTZ black hole geometry. We find that the critical time $t_{c}$ is shorter than that in the BTZ case. This is roughly because the deformation enlarges the wormhole region and so increases the connected surface area. In the black hole geometry with a sufficiently large deformation parameter, we find that the

\footnotetext{
${ }^{1}$ There is also a static type of Janus deformation of BTZ black hole [20].
} 
area of the disconnected one becomes always smaller and that the holographic entanglement entropy is already proportional to the size of the subsystem from the beginning, unlike the BTZ case.

This paper is organized as follows. In section 2, we review properties of the threedimensional Janus black hole with emphasis on the difference from the BTZ black hole. In section 3, we compute the area of extremal surfaces with appropriate boundary conditions in this black hole geometry. In section 4 we discuss the time evolution of the holographic entanglement entropy. We conclude this paper in section 5 .

\section{Properties of three-dimensional Janus Black Hole}

Here we summarize the properties of the three dimensional time-dependent Janus black hole with emphasis on its causal structure and dual CFT interpretation.

\subsection{The three-dimensional Janus metric}

\subsubsection{Time-dependent Janus deformation of BTZ metric}

The metric of the Janus black hole with its horizon radius $L r_{0}$ is given by

$$
d s^{2}=L^{2} \frac{d \mu^{2}-d \tau^{2}+r_{0}^{2} \cos ^{2} \tau d \theta^{2}}{g(\mu)^{2}},
$$

where the only dimensionful quantity is the AdS radius $L$. The conformal factor $g(\mu)$ is defined as

$$
g(\mu)=\frac{\operatorname{cn}\left(\kappa_{+} \mu, k^{2}\right)}{\kappa_{+} \operatorname{dn}\left(\kappa_{+} \mu, k^{2}\right)} \quad \kappa_{ \pm}:=\sqrt{\frac{1 \pm \sqrt{1-2 \gamma^{2}}}{2}} \quad k:=\frac{\kappa_{-}}{\kappa_{+}} .
$$

This metric is a one-parameter generalization of the BTZ black hole metric by "Janus deformation parameter" $0 \leq \gamma<1 / \sqrt{2}$. When $\gamma=0$, the factor $g(\mu)$ becomes $\cos \mu$ and then the metric reduces to the BTZ metric, with its inverse temperature

$$
\beta=\frac{2 \pi}{r_{0}},
$$

in the unit of the AdS radius $L$. The conformal boundaries $g(\mu)=0$ are located at $\mu= \pm \mu_{0}$, where $\mu_{0}:=K\left(k^{2}\right) / \kappa_{+}$and $K\left(k^{2}\right)$ is the complete elliptic integral of the 1st kind $K\left(k^{2}\right):=\int_{0}^{\pi / 2} d \theta / \sqrt{1-k^{2} \sin ^{2} \theta}$.

\subsubsection{Dual CFT coordinate $(t, \theta)$ and UV cutoff $\epsilon_{\mathrm{CFT}}$}

In applying AdS/CFT techniques, another time coordinate $\tanh r_{0} t:=\sin \tau$ is useful, because the flat metric $-d t^{2}+d \theta^{2}$ of the dual CFT becomes manifest:

$$
d s^{2}=L^{2}\left[d y^{2}+\frac{r_{0}^{2}}{\tilde{g}(y)^{2} \cosh ^{2} r_{0} t}\left(-d t^{2}+d \theta^{2}\right)\right] .
$$


Here we have also replaced the radial coordinate $\mu$ with another one $y$ such that $\tanh y=$ $\operatorname{sn}\left(\kappa_{+} \mu, k^{2}\right)$, measuring the proper length $d y=d \mu / g(\mu)$, and we have rewritten the factor $g(\mu)$ as

$$
\tilde{g}(y):=g(\mu(y))=\frac{1}{\kappa_{+} \sqrt{\left(1-k^{2}\right) \cosh ^{2} y+k^{2}}}=\sqrt{\frac{2}{1+\sqrt{1-2 \gamma^{2}} \cosh 2 y}} .
$$

In this coordinate $y$, the origin $\mu=0$ corresponds to $y=0$ and the conformal boundaries $\mu= \pm \mu_{0}$ are located at $y \rightarrow \pm \infty$.

Near the conformal boundaries $y \rightarrow \pm \infty$, the metric (2.4) approaches to the pure AdS metric in Poincaré coordinate

$$
d s^{2}=L^{2} \frac{d z^{2}-d t^{2}+d \theta^{2}+\mathcal{O}(z)}{z^{2}}
$$

with the following identification

$$
z:=\frac{2}{\sqrt[4]{1-2 \gamma^{2}} r_{0}} e^{-|y|} \cosh r_{0} t
$$

Hence the CFT UV cutoff $\epsilon_{\mathrm{CFT}}$ is given as

$$
\epsilon_{\mathrm{CFT}}=\frac{2}{\sqrt[4]{1-2 \gamma^{2}} r_{0}} e^{-y_{\infty}} \cosh r_{0} t_{\infty},
$$

where $y_{\infty}(\gg 1)$ is a bulk volume regulator and $t_{\infty}$ is the time $t$ in the CFT at $y= \pm y_{\infty}$.

\subsubsection{As a solution of Einstein-scalar theory}

This geometry is a solution of the three-dimensional Einstein-scalar system

$$
S=\frac{1}{16 \pi G} \int d^{3} x \sqrt{g}\left(R-g^{a b} \partial_{a} \phi \partial_{b} \phi+\frac{2}{L^{2}}\right),
$$

with a scalar field configuration

$$
\begin{aligned}
\phi & =\phi_{0}+\sqrt{2}\left(\tanh ^{-1}\left(k \operatorname{sn}\left(\kappa_{+} \mu, k^{2}\right)\right)+\log \sqrt{1-k^{2}}\right) \\
& =\phi_{0}+\sqrt{2}\left(\tanh ^{-1}(k \tanh y)+\log \sqrt{1-k^{2}}\right) .
\end{aligned}
$$

Note that the scalar field value $\phi_{+}:=\phi(y=\infty)$ on the right boundary is different from the one $\phi_{-}:=\phi(y=-\infty)$ on the left boundary by

$$
\phi_{+}-\phi_{-}=2 \sqrt{2} \tanh ^{-1} k=\sqrt{2} \tanh ^{-1} \sqrt{2} \gamma .
$$

This three-dimensional system can be embedded in type IIB supergravity in ten dimensions with an appropriate ansatz [1]. Then in the same way as the standard D1-D5 black hole [24-26], the boundary CFTs are given by the IR fixed points of the two-dimensional $\mathcal{N}=(4,4)$ supersymmetric $\mathrm{SU}\left(N_{1}\right) \times \mathrm{SU}\left(N_{5}\right)$ quiver field theories, which turn out to be $\sigma$-models on the instanton moduli space $\mathcal{M}=M_{4}^{N_{1} N_{5}} / S_{N_{1} N_{5}}$. The bulk scalar field $\phi$ is 
identified with the dilaton, and hence the boundary values $\phi_{ \pm}$are related to the coupling constants $g_{ \pm}$of those boundary quiver theories [1]. In terms of the IR $\sigma$-models, this difference in the boundary values leads to the difference in the overall coefficients of the actions on the two boundaries.

Although the difference between $\phi_{+}$and $\phi_{-}$(2.11) becomes very large when we take $\gamma$ very close to $1 / \sqrt{2}$, we can also take $\phi_{0}$ negatively large so that classical gravity does not break down. In terms of the dual boundary theory, it requires that the theory is weakly coupled in the sense of the Yang-Mills couplings whereas it is strongly coupled in the viewpoint of the 't Hooft couplings, as usual.

\subsection{Main differences from BTZ black hole}

\subsubsection{Causal shadow region}

By using the conformally flat $(\mu, \tau)$ coordinate $(2.1)$, one can draw the Penrose diagram of the time-dependent Janus black hole geometry (see Figure 1). The diagram is horizontally longer than that of the BTZ geometry, because the width $2 \mu_{0}=2 K\left(k^{2}\right) / \kappa_{+}$in the $\mu$ coordinate between the two conformal boundaries monotonically increases with the deformation parameter $\gamma$.

As a consequence, unlike the BTZ geometry $(\gamma=0)$, the three-dimensional Janus black hole geometry $(\gamma>0)$ has a finite region causally disconnected from the both conformal boundaries $\mu= \pm \mu_{0}$. Such regions are sometimes called "causal shadow" [14, 15]. It is an interesting question how the dual CFT encodes information on causal shadow regions. As a first step to answer this question, we will compute holographic entanglement entropies in the Janus black hole geometry in the next two sections, because holographic entanglement entropies can be affected by the inside of the causal shadow.

\subsubsection{Time-dependence}

Unlike the BTZ metric $(\gamma=0)$, the Janus metric $(\gamma>0)$ is time-dependent, that is to say, has no timelike Killing vector. As a result, its apparent horizon

$$
\tan \tau=-\frac{d}{d \mu} \log g(\mu)
$$

in a time slice $\tau=$ const. becomes different from the event horizon $\tau-\pi / 2=\mu-\mu_{0}$ (See Figure 1).

\subsection{The CFT interpretation of the Janus black hole}

When $\gamma=0$, the Janus black hole reduces to the ordinary eternal BTZ black hole, which is dual to the thermofield double state $[22,23]$

$$
|\Psi\rangle=\frac{1}{\sqrt{Z}} \sum_{n} e^{-\frac{\beta}{2} E_{n}}\left|E_{n}\right\rangle\left|E_{n}\right\rangle .
$$

The inverse temperature $\beta$ is given by (2.3). 


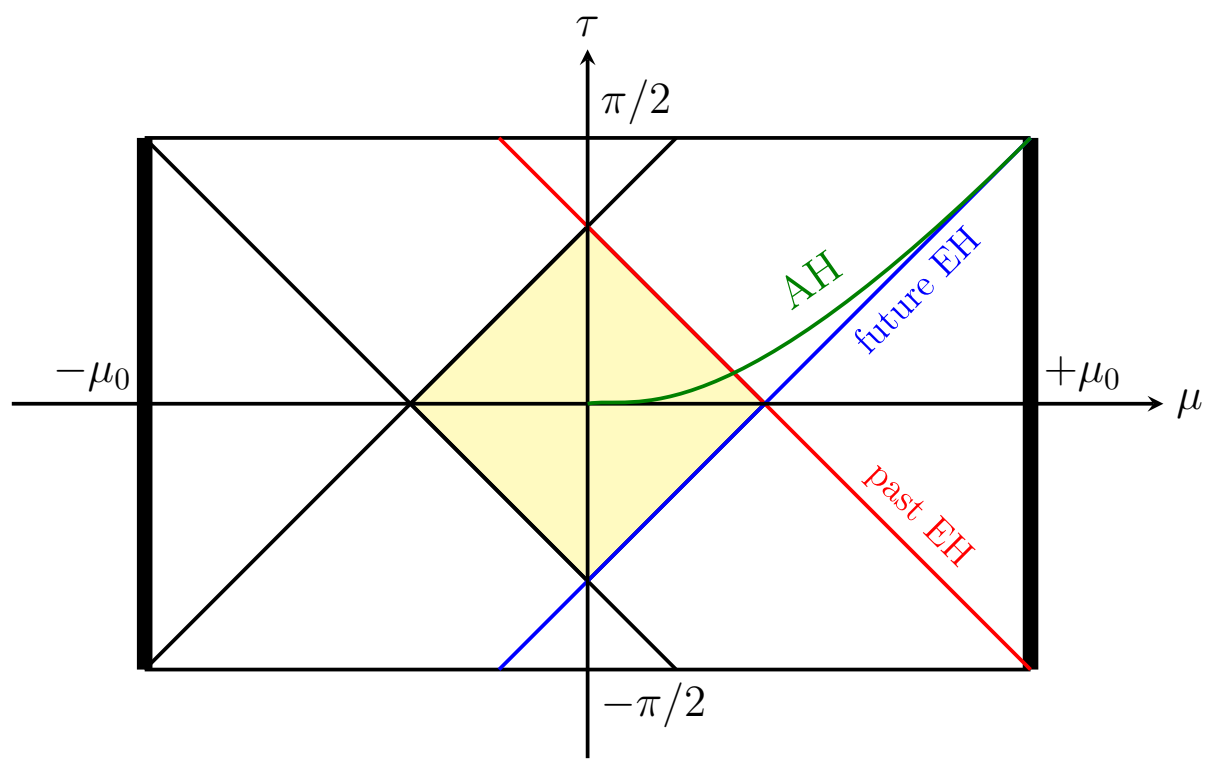

Figure 1. Penrose diagram of the three-dimensional time-dependent Janus black hole. The two conformal boundaries are located at $\mu= \pm \mu_{0}$ (thick lines), and the diagram is a wide rectangle because $\mu_{0} \geq \pi / 2$. The blue and red lines represent, respectively, the future and past event horizons which intersect with the right hand side boundary. The yellow shaded region corresponds to the "causal shadow" region, which is causally disconnected from the both boundaries. The apparent horizons (green line) in time slices $\tau=$ const. are located inside the future event horizon.

If we turn on the parameter $\gamma$, the Hamiltonian $H_{+}$on the right boundary and $H_{-}$on the left boundary become different, as was explained in section 2.1.3. Hence it is natural to conjecture [21] that the Janus black hole is dual to a state

$$
|\Psi\rangle=\frac{1}{\sqrt{Z}} \sum_{(m, n)} e^{-\frac{\beta}{4}\left(E_{n}^{-}+E_{m}^{+}\right)}\left\langle E_{m}^{+} \mid E_{n}^{-}\right\rangle\left|E_{m}^{+}\right\rangle\left|E_{n}^{-}\right\rangle .
$$

This conjecture has passed some nontrivial checks. For example, the one point function of the Lagrangian density was computed both on gravity and CFT sides, which agrees up to the second order in $\gamma[21]$.

\section{Calculation of holographic entanglement entropy}

In this section, we compute a holographic entanglement entropy on the three-dimensional Janus black hole geometry to study an entanglement between the left and right CFT's. We take our subsystem $A$ to be two disjoint same intervals $-\theta_{\infty} \leq \theta \leq \theta_{\infty}$ in each of the left and right CFT at a fixed time $t=t_{\infty}$ (see Figure 2).

\subsection{Covariant holographic entanglement entropy}

It has been conjectured [8] that for a given bulk geometry, the entanglement entropy of the dual CFT state is given by the area of the extremal surface ${ }^{2}$ in the bulk which are

\footnotetext{
${ }^{2}$ The original holographic entanglement entropy formula [6, 7] (later proven in [27]) with minimal surface prescription is only applicable to static bulk geometries. The extension (3.1) to general geometries is
} 

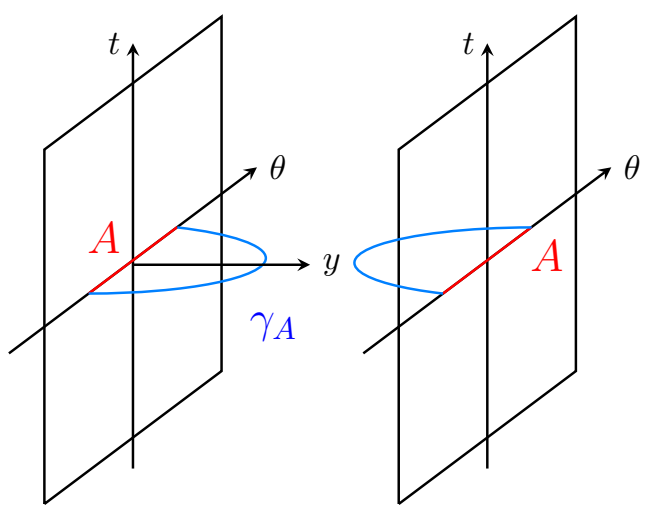

(a) disconnected phase

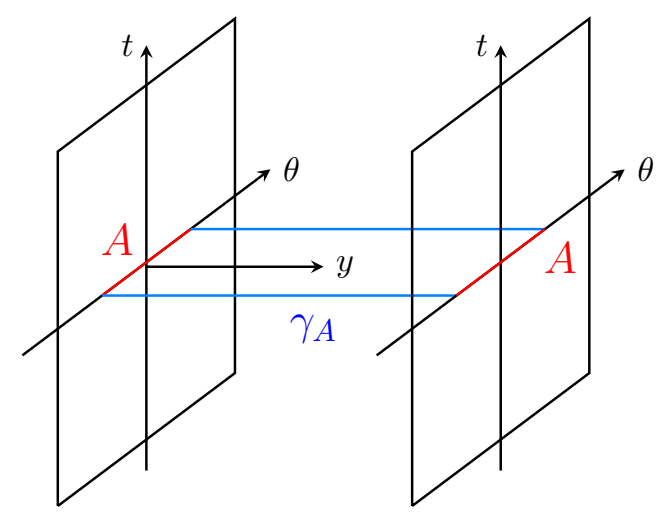

(b) connected phase

Figure 2. The subsystem $A$ (two red lines) is taken as two disjoint intervals of the same length $\Delta \theta=2 \theta_{\infty}$ in the right and left boundary (two black squares). The extremal surface $\gamma_{A}$ (blue lines) has two phases: disconnected phase $(a)$ and connected phase $(b)$.

anchored to $\partial A$ in the conformal boundary,

$$
S_{A}=\operatorname{ext} \frac{A\left(\gamma_{A}\right)}{4 G_{N}},
$$

where $G_{N}$ is the three-dimensional Newtonian constant. The extrema is chosen among the surfaces $\gamma_{A}$ which are homologous to the subsystem $A$ and satisfying $\partial A=\partial \gamma_{A}$. If there are multiple extremal surfaces, we should choose the one with the minimum area among them.

In the current setup with the subsystem $A=\left\{\left( \pm y_{\infty}, t_{\infty}, \theta\right) ;-\theta_{\infty} \leq \theta \leq \theta_{\infty}\right\}$ in the Janus black hole geometry (2.4), the extremal surface can take two types of topologies (see Figure 2), "connected phase" and "disconnected phase", like the usual BTZ black holes [9]. The disconnected type consists of two geodesics which start from and end at the same boundary (see Figure $2(a))$; starting from $\left( \pm y_{\infty}, t_{\infty},-\theta_{\infty}\right)$, turning around at $\left( \pm y_{*}, t_{*}, 0\right)$ and ending at $\left( \pm y_{\infty}, t_{\infty}, \theta_{\infty}\right)$. The connected type consists of two geodesics which connect the two boundaries (see Figure $2(b))$; starting from $\left(y_{\infty}, t_{\infty}, \pm \theta_{\infty}\right)$ and ending at $\left(-y_{\infty}, t_{\infty}, \pm \theta_{\infty}\right)$.

In the following, we will obtain and solve differential equations for each type of extremal surfaces. Identifying the area functional

$$
A[t(y), \theta(y)]=L \int d y \sqrt{1+\frac{r_{0}^{2}}{\tilde{g}(y)^{2} \cosh ^{2} r_{0} t}\left(-\dot{t}^{2}+\dot{\theta}^{2}\right)}
$$

with a classical action for dynamical variables $t(y)$ and $\theta(y)$ as for "time" $y$, this problem reduces to just an Euler-Lagrange problem. Here the dot $\left(^{\circ}\right)$ represents the "time" deriva-

achieved by just replacing the "minimum" on the time-slice by the "extremum" in the spacetime.

There are many equivalent constructions which look different. The extremal surface explained above (called $\mathcal{W}$ in the original paper [8]), the surface with vanishing traces of extrinsic curvatures $\left(\mathcal{Y}_{\text {ext }}\right.$ ), and a surface constructed by using light-sheets $\left(\mathcal{Y}_{\mathcal{A}_{t}}^{\min }\right)$, are eventually all equivalent. See the original paper for the detail. 
tive $d / d y$. We will see that the disconnected surface, as well as the connected one, can penetrate the event horizon, and both of their areas are dependent on the boundary time $t_{\infty}$. The phase transition between these two types will be discussed in section 4 .

\subsection{Extremal areas in connected phase}

For connected surfaces, the area functional is extremized when $\theta=$ const. $\left(= \pm \theta_{\infty}\right)$. Then the "action" (3.2) becomes

$$
A[t(y)] / L=\int_{-y_{\infty}}^{y_{\infty}} d y \sqrt{1-\frac{r_{0}^{2} \dot{t}^{2}}{\tilde{g}(y)^{2} \cosh ^{2} r_{0} t}},
$$

for each of the two pieces of the surface $\left(\theta= \pm \theta_{\infty}\right)$. Here $y_{\infty}$ is the bulk volume regulator, which also regulates the area functional. This functional has one conserved charge $E$ :

$$
\begin{aligned}
E:=\frac{\delta A / L}{\delta \dot{t}} & =\frac{-r_{0}^{2} \dot{t}}{\tilde{g}(y) \cosh r_{0} t \sqrt{\tilde{g}(y)^{2} \cosh ^{2} r_{0} t-r_{0}^{2} \dot{t}^{2}}} \\
& \Leftrightarrow \quad \dot{t}=\frac{-E \tilde{g}(y)^{2} \cosh ^{2} r_{0} t}{\sqrt{r_{0}^{2}+E^{2} \tilde{g}(y)^{2} \cosh ^{2} r_{0} t}},
\end{aligned}
$$

associated to its $t$-translation symmetry. But this charge $E$ vanishes, because $\dot{t}$ cannot change its sign and we have the boundary condition $\int_{-y_{\infty}}^{y_{\infty}} \dot{t} d y=t_{\infty}-t_{\infty}=0$. In the result, the total area of the connected extremal surface can be explicitly calculated as

$$
\begin{aligned}
A_{c}\left(t_{\infty}, \theta_{\infty}\right) / L & =2 \times 2 y_{\infty} \\
& =4 \log \frac{2 \cosh r_{0} t_{\infty}}{r_{0} \epsilon_{\mathrm{CFT}}}-\log \left(1-2 \gamma^{2}\right) .
\end{aligned}
$$

To derive this, we used the relation between the regulator $y_{\infty}$ and the CFT cutoff $\epsilon_{\mathrm{CFT}}$ (2.8). For later purposes, it is convenient to define the notion of "renormalized" area which is given by

$$
\begin{aligned}
A_{c}^{(\text {ren })} / L & \equiv A_{c}\left(t_{\infty}, \theta_{\infty}\right) / L+4 \log \epsilon_{\mathrm{CFT}} \\
& =4 \log \frac{2 \cosh r_{0} t_{\infty}}{r_{0}}-\log \left(1-2 \gamma^{2}\right) .
\end{aligned}
$$

Note that the connected surface area becomes arbitrarily large in $\gamma^{2} \rightarrow \frac{1}{2}$ limit. This illuminates the fact that the length of the wormhole behind the Janus black hole becomes infinitely long in this limit.

\subsection{How to calculate extremal areas in disconnected phase}

In this subsection, we represent the area of the disconnected surfaces as a function of boundary coordinates $\left(t_{\infty}, \theta_{\infty}\right)$. The disconnected surfaces consist of two disjoint geodesics, one of which is located in the right region $y>0$ and the other is in the left region $y<0$. In what follows, we take the $y>0$ part of the surfaces, because the $y<0$ part can be identified with $y>0$ part by the parity transformation $y \rightarrow-y$. 
The disconnected surface area $A_{d c}$ can be given by an integral from the boundary $y=$ $y_{\infty}$ to the returning point $y=y_{*}$, at which the derivative $\dot{\theta}$ of the surface $(t, \theta)=(t(y), \theta(y))$ diverges. Its location $\left(y_{*}, t_{*}\right)$ is determined by the boundary coordinates $\left(t_{\infty}, \theta_{\infty}\right)$, and so we can represent the area $A_{d c}$ as a function of $\left(t_{\infty}, \theta_{\infty}\right)$ by substituting the expression $y_{*}=y_{*}\left(t_{\infty}, \theta_{\infty}\right)$ into the area integral.

\subsubsection{Solving the equation of motion}

We can solve the Euler-Lagrange equations for $t(y)$ and $\theta(y)$ in the following way.

The action (3.2) has one conserved charge $J$,

$$
J:=\frac{\delta A / L}{\delta \dot{\theta}}=\frac{1}{\tilde{g}(y) \cosh r_{0} t} \frac{r_{0}^{2} \dot{\theta}}{\sqrt{\tilde{g}(y)^{2} \cosh ^{2} r_{0} t+r_{0}^{2}\left(-\dot{t}^{2}+\dot{\theta}^{2}\right)}}
$$

associated to its $\theta$ translation symmetry. This charge $J$ can be expressed by the returning point location $\left(y_{*}, t_{*}\right)$ as $J=r_{0}\left(\tilde{g}\left(y_{*}\right) \cosh r_{0} t_{*}\right)^{-1}$, because $\dot{\theta}$ in (3.7) diverges at the returning point. With the aid of this constant charge $J$, the equation of motion for $t(y)$ can be rewritten into an equation for $t(\theta)$ without any $\tilde{g}(y)$ dependence:

$$
\begin{aligned}
\frac{d}{d y} \frac{\delta A}{\delta \dot{t}}-\frac{\delta A}{\delta t}=0 & \Leftrightarrow \frac{d}{d y}\left(J \frac{\dot{t}}{\dot{\theta}}\right)=J r_{0} \frac{-\dot{t}^{2}+\dot{\theta}^{2}}{\dot{\theta}} \tanh r_{0} t \\
& \Leftrightarrow \frac{d^{2} t}{d \theta^{2}}=r_{0}\left[1-\left(\frac{d t}{d \theta}\right)^{2}\right] \tanh r_{0} t
\end{aligned}
$$

whose general solution is given by $\sinh r_{0} t=\sinh A \cosh r_{0}(\theta+B)$ with some constants $A, B$. These constants $A, B$ are determined by geometrical conditions $\left.\theta\right|_{y=y_{*}}=0$ and $d t /\left.d \theta\right|_{y=y_{*}}=0$ as

$$
\sinh r_{0} t=\sinh r_{0} t_{*} \cosh r_{0} \theta
$$

This relation allows us to erase $\theta$ in (3.7), yielding a 1st order differential equation of $t$ :

$$
\dot{t}=\frac{\cosh r_{0} t}{r_{0} \tilde{g}\left(y_{*}\right) \cosh r_{0} t_{*}} \sqrt{\frac{\cosh ^{2} r_{0} t-\cosh ^{2} r_{0} t_{*}}{1-\left(\tilde{g}(y) / \tilde{g}\left(y_{*}\right)\right)^{2}}}
$$

which has a unique solution

$$
\sqrt{1-\frac{\sinh ^{2} r_{0} t_{*}}{\sinh ^{2} r_{0} t}}(=\tanh \theta)=\cosh r_{0} t_{*} \tanh \left[\int_{y_{*}}^{y} d y \frac{\tilde{g}(y)^{2}}{\sqrt{\tilde{g}\left(y_{*}\right)^{2}-\tilde{g}(y)^{2}}}\right],
$$

with an initial condition $t\left(y_{*}\right)=t_{*}$. This expression gives the unique solution $(t(y), \theta(y))$ of the equations of motion, in terms of the returning point location $\left(y_{*}, t_{*}\right)$.

\subsubsection{Returning point $\left(y_{*}, t_{*}\right)$}

The boundary condition $\left(t\left(y_{\infty}\right)=t_{\infty}, \theta\left(y_{\infty}\right)= \pm \theta_{\infty}\right)$ determines $t_{*}$ by (3.10) as

$$
\sinh r_{0} t_{*}=\frac{\sinh r_{0} t_{\infty}}{\cosh r_{0} \theta_{\infty}},
$$




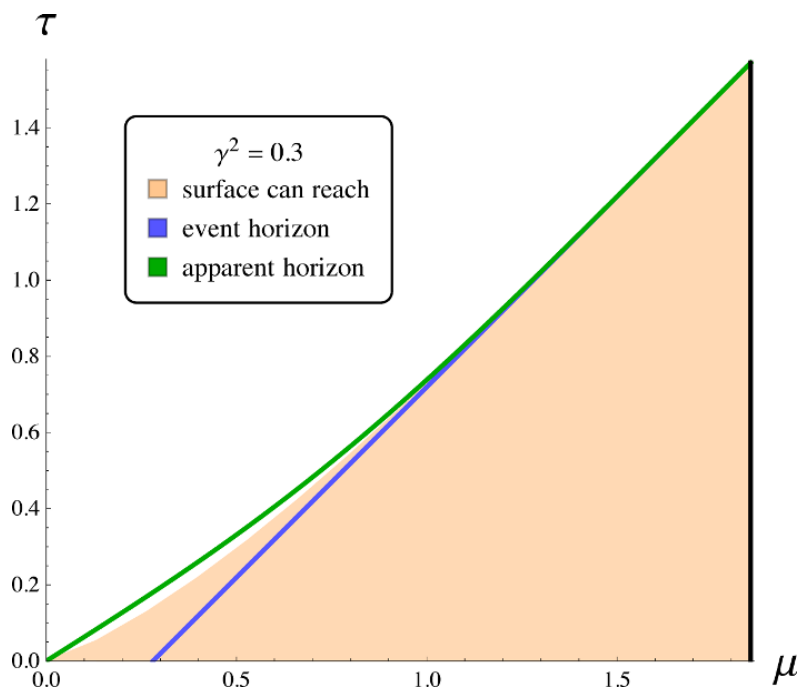

Figure 3. How deeply the extremal surfaces in the disconnected phase can go inside the Janus black hole (with $\gamma^{2}=0.3$ in the figure). The shaded orange region represents where the extremal surfaces can pass through. The extremal surfaces can go beyond the event horizon (blue line), but cannot go beyond the apparent horizon (green line).

and $y_{*}$ by $(3.10)$ and $(3.12)$ as

$$
\sinh \left[\int_{y_{*}}^{y_{\infty}} d y \frac{\tilde{g}(y)^{2}}{\sqrt{\tilde{g}\left(y_{*}\right)^{2}-\tilde{g}(y)^{2}}}\right]=\frac{\sinh r_{0} \theta_{\infty}}{\cosh r_{0} t_{\infty}} .
$$

Note that there are bulk points which cannot be returning points for any boundary value $\left(t_{\infty}, \theta_{\infty}\right)$, and that there exists a region which cannot be reached by the connected surfaces (see figure 3). It might be interesting that the surface can go beyond the event horizon but cannot go beyond the apparent horizon.

\subsubsection{Extremal surface area}

Plugging (3.10) and (3.11) into the definition of the surface area (3.2), we obtain the disconnected extremal surface area

$$
A_{d c}\left(t_{\infty}, \theta_{\infty}\right) / L=4 \int_{y_{*}}^{y_{\infty}} d y \frac{\tilde{g}\left(y_{*}\right)}{\sqrt{\tilde{g}\left(y_{*}\right)^{2}-\tilde{g}(y)^{2}}},
$$

as a function of $\left(t_{\infty}, \theta_{\infty}\right)$, with $y_{*}$ implicitly determined by $\left(t_{\infty}, \theta_{\infty}\right)$ through (3.14).

Note that this area has a UV divergence $-4 \log \epsilon_{\mathrm{CFT}}$, because $\tilde{g}(y) \rightarrow 0$ at the each boundary and

$$
A_{d c} / L \rightarrow 4 \int^{y_{\infty}} d y \sim 4 y_{\infty}=4 \log \frac{2 \cosh r_{0} t_{\infty}}{\sqrt[4]{1-2 \gamma^{2}} r_{0} \epsilon_{\mathrm{CFT}}} .
$$

This UV divergence can be renormalized as

$$
\begin{aligned}
A_{d c}^{(\mathrm{ren})} / L & \equiv A_{d c} / L+4 \log \epsilon_{\mathrm{CFT}} \\
& =A_{d c} / L-\log \left(1-2 \gamma^{2}\right)+4 \log \frac{2 \cosh r_{0} t_{\infty}}{r_{0}}-4 y_{\infty} .
\end{aligned}
$$




\subsection{Some limits of extremal surface areas in disconnected phase}

It is generally difficult to calculate the area of the disconnected surface. In this subsection, we address some limits in which this area is explicitly calculable. In sections 3.4.1 and 3.4.2, we compute the disconnected surface area $A_{d c}^{(\text {ren })}\left(t_{\infty}, \theta_{\infty}\right)$ with a large subsystem $\left(\theta_{\infty} \gg\right.$ $\left.r_{0}^{-1}\right)$, in the early time $\left(t_{\infty} \ll \theta_{\infty}\right)$ and late time $\left(t_{\infty} \gg \theta_{\infty}\right)$ limit. In section 3.4.3, we compute the area in the small $\gamma$ limit.

\subsubsection{Early time limit for large subsystem $\left(\theta_{\infty} \gg t_{\infty}\right)$}

In this parameter region, the returning point $\left(y_{*}, t_{*}\right)$ is close to the origin $(0,0)$, which can be seen as follows. The $t_{*}$ is determined by (3.13) as

$$
r_{0} t_{*} \simeq 2 e^{-r_{0} \theta_{\infty}} \sinh r_{0} t_{\infty} \quad(\ll 1),
$$

where we used $\cosh r_{0} \theta_{\infty} \simeq e^{r_{0} \theta_{\infty}} / 2$ and $\sinh r_{0} t_{*} \simeq r_{0} t_{*}$. The $y_{*}$ is determined by (3.14) as

$$
r_{0} \theta_{\infty}-\log \cosh r_{0} t_{\infty} \simeq \int_{y_{*}}^{y_{\infty}} d y \frac{\tilde{g}(y)^{2}}{\sqrt{\tilde{g}\left(y_{*}\right)^{2}-\tilde{g}(y)^{2}}} \quad(\gg 1) .
$$

This means $y_{*} \ll 1$, because the left hand side of (3.19) is large while the integral of the right hand side is a monotonically decreasing function of $y_{*}$, diverging at $y_{*} \rightarrow 0$. In fact, the right hand side integral can be evaluated as

$$
r_{0} \theta_{\infty}-\log \cosh r_{0} t_{\infty} \simeq-\frac{1}{\sqrt{\kappa_{+}^{2}-\kappa_{-}^{2}}} \log \left[\frac{\kappa_{+}+\sqrt{\kappa_{+}^{2}-\kappa_{-}^{2}}}{4} y_{*}\right]
$$

in the limit $y_{*} \rightarrow 0$, by changing the integration variable from $y$ to $z:=\tanh y$.

By solving this for $y_{*}$ and plugging it into (3.17), we obtain the renormalized area $A_{d c}^{(\mathrm{ren})}\left(t_{\infty}, \theta_{\infty}\right)$ as a function of $\left(t_{\infty}, \theta_{\infty}\right)$. This can be carried out by evaluating the integration of (3.15) similarly as

$$
\begin{aligned}
\int_{y_{*}}^{y_{\infty}} d y & \frac{\tilde{g}\left(y_{*}\right)}{\sqrt{\tilde{g}\left(y_{*}\right)^{2}-\tilde{g}(y)^{2}}} \\
& \simeq-\frac{\kappa_{+}}{\sqrt{\kappa_{+}^{2}-\kappa_{-}^{2}}} \log \left[\frac{\kappa_{+}+\sqrt{\kappa_{+}^{2}-\kappa_{-}^{2}}}{4} y_{*}\right]-\log \left[\frac{\kappa_{+}+\sqrt{\kappa_{+}^{2}-\kappa_{-}^{2}}}{\sqrt{\kappa_{+}^{2}-\kappa_{-}^{2}}}\right]+y_{\infty}
\end{aligned}
$$

in the limit $y_{*} \rightarrow 0$. We can delete $y_{*}$ by (3.20), which results in

$$
A_{d c}^{(\mathrm{ren})}\left(t_{\infty}, \theta_{\infty}\right) / L \simeq 4 \kappa_{+} r_{0} \theta_{\infty}+4\left(1-\kappa_{+}\right) \log \cosh r_{0} t_{\infty}-4 \log \left[\frac{\kappa_{+}+\sqrt{\kappa_{+}^{2}-\kappa_{-}^{2}}}{2} r_{0}\right]
$$

Note that the area linearly grows with both $t_{\infty}$ and $\theta_{\infty}$ with different coefficients, when $\theta_{\infty} \gg t_{\infty} \gg r_{0}^{-1}$. 


\subsubsection{Late time limit for large subsystem $\left(t_{\infty} \gg \theta_{\infty} \gg r_{0}^{-1}\right)$}

In this parameter region, (3.13) and (3.14) lead to

$$
2 e^{-r_{0} t_{*}} \simeq \int_{y_{*}}^{y_{\infty}} \frac{\tilde{g}(y)^{2} d y}{\sqrt{\tilde{g}\left(y_{*}\right)^{2}-\tilde{g}(y)^{2}}} \simeq e^{r_{0}\left(\theta_{\infty}-t_{\infty}\right)} \quad(\ll 1)
$$

where we used $\sinh x \simeq \cosh x \simeq e^{x} / 2$ for $x \gg 1$ and $\sinh x \simeq x$ for $x \ll 1$. This in turn implies $y_{*} \gg 1$, therefore the integrals in (3.23) and (3.15) can be respectively approximated as

$$
\begin{aligned}
& \int_{y_{*}}^{y_{\infty}} \frac{\tilde{g}(y)^{2} d y}{\sqrt{\tilde{g}\left(y_{*}\right)^{2}-\tilde{g}(y)^{2}}} \simeq \frac{2}{\sqrt[4]{1-2 \gamma^{2}}} e^{-y_{*}}, \\
& \int_{y_{*}}^{y_{\infty}} \frac{\tilde{g}\left(y_{*}\right) d y}{\sqrt{\tilde{g}\left(y_{*}\right)^{2}-\tilde{g}(y)^{2}}} \simeq y_{\infty}-y_{*}+\log 2
\end{aligned}
$$

where we also used $y_{\infty}-y_{*} \gg 1$. By substituting (3.25) into (3.15), and by erasing $y_{\infty}$ and $y_{*}$ with the aid of (2.8), (3.23) and (3.24), we can evaluate the renormalized area $A_{d c}^{(\mathrm{ren})}(3.17)$ as

$$
A_{d c}^{(\mathrm{ren})}\left(t_{\infty}, \theta_{\infty}\right) / L \simeq 4\left(r_{0} \theta_{\infty}-\log r_{0}\right)
$$

which does not depend on either of $\gamma$ or $t_{\infty}$. Then in particular, it coincides with the BTZ $\left(\gamma^{2}=0\right)$ result.

\subsubsection{Up to the lowest order of $\gamma^{2}$}

So far, we have seen the early time $t_{\infty} \ll \theta_{\infty}$ and the late time $\theta_{\infty} \ll t_{\infty}$ behavior of the disconnected surface area for general $\gamma$, but the surface phase transition discussed in the next section typically occurs at the intermediate time region $t_{\infty} \sim \theta_{\infty}$. To obtain an analytic expression applicable to the whole time region, let us evaluate the surface area up to the lowest order of the deformation $\gamma^{2}$. By expanding the relation (3.14) and the area integral (3.15) up to the order of $\gamma^{2}$, we get

$$
A_{d c}^{(r e n)} / L=4 \log \left(\frac{2}{r_{0}} \sinh r_{0} \theta\right)-\left(\frac{3 F^{2}+2}{2 \sqrt{1+F^{2}}} \operatorname{coth}^{-1}\left(\sqrt{1+F^{2}}\right)-\frac{3}{2}\right) \gamma^{2}+\mathcal{O}\left(\gamma^{4}\right),
$$

where

$$
F(t, \theta)=\frac{\cosh r_{0} t_{\infty}}{\sinh r_{0} \theta_{\infty}}
$$

The detail of this calculation is explained in appendix A. Note that when $\gamma=0$, it reduces to the usual thermal result. In the early $(F \ll 1)$ and late $(F \gg 1)$ time limits, it respectively reproduces the results (3.22) and (3.26) in the previous section. 


\section{Time evolution of entanglement entropy and phase transition}

Here we discuss the time-dependent behavior of the holographic entanglement entropy. Since there are two extremal surfaces in the bulk geometry, the holographic entanglement entropy $S_{A}$ is given by choosing the one with the minimum area among them,

$$
S_{A}=\frac{1}{4 G_{N}} \min \left\{A_{c}, A_{d c}\right\},
$$

where $A_{c}$ and $A_{d c}$ are given by (3.5) and (3.15) respectively. As we will see below, the entropy $S_{A}$ behaves very differently depending on the deformation parameter $\gamma$.

$\gamma=\mathbf{0}$. When $\gamma=0$, the spacetime reduces to the BTZ black hole, and does not contain any causal shadow region. The connected and disconnected surface areas are respectively given by

$$
A_{c}\left(t_{\infty}, \theta_{\infty}\right) / L=4 \log \left(\frac{2 \cosh r_{0} t_{\infty}}{r_{0} \epsilon_{\mathrm{CFT}}}\right), \quad A_{d c}\left(t_{\infty}, \theta_{\infty}\right) / L=4 \log \left(\frac{2 \sinh r_{0} \theta_{\infty}}{r_{0} \epsilon_{\mathrm{CFT}}}\right) .
$$

Let us take a sufficiently large subsystem $r_{0} \theta_{\infty} \gg 1$. The entropy initially grows linearly with time because the connected surface is chosen in accordance with $A_{c}<A_{d c}$, and stops growing at a critical time $t_{\infty}=t_{c} \simeq \theta_{\infty}$. After the critical time, it ends up with a constant value, double the value of the thermal entropy, because the disconnected surface becomes chosen in accordance with $A_{d c}<A_{c}$.

This time-dependent behavior such as the sharp phase transition can be also be observed on the CFT side, since the time-scale of the transition is given by $\beta$ [9] and now $r_{0} \theta \gg 1$ implies $t_{c} \gg \beta$. Furthermore, the initial entanglement entropy at $t_{\infty}=0$ can be identified with the contribution from the boundary of $A$ (4 points). The time-dependent behavior can be intuitively understood in the so-called quasi-particle picture [10]. In this picture, we assume that a pair creation of entangled quasi-particles occurs at every spatial point at the initial time, and that the pair propagate in opposite directions at the speed of light. A pair contributes to the entanglement entropy if one of the pair is inside the subsystem and the other of the pair is outside the subsystem. This picture correctly reproduces the linear growth and saturation of the entanglement entropy.

$\mathbf{0}<\gamma^{2} \ll \frac{1}{2}$. When $0<\gamma^{2} \ll \frac{1}{2}$, the story is quite similar to the BTZ case, $\gamma=0$. The entanglement entropy grows up until a critical time $t_{\infty}=t_{c} \simeq \theta_{\infty}$, when the areas of the two surfaces become equal and a phase transition takes place. At that time, the growth rate of the entanglement entropy suddenly decreases discontinuously, but does not immediately become zero, unlike the BTZ case. The entanglement entropy continues to grow very slowly and converges to a constant independent of $\gamma$. Hence the final value is identical with that of the BTZ case, $\gamma=0$, in particular.

Another important difference from the BTZ black hole case is that the initial entanglement entropy includes an additional positive term $\left(-\frac{L}{4 G_{N}} \log \left(1-2 \gamma^{2}\right)\right)$. This term can be regarded as a kind of boundary entropy, which is the contribution of defects in the system [28] (see also [29] for the holographic realization). Note that in our system the defect is localized along the Euclidean time direction. 


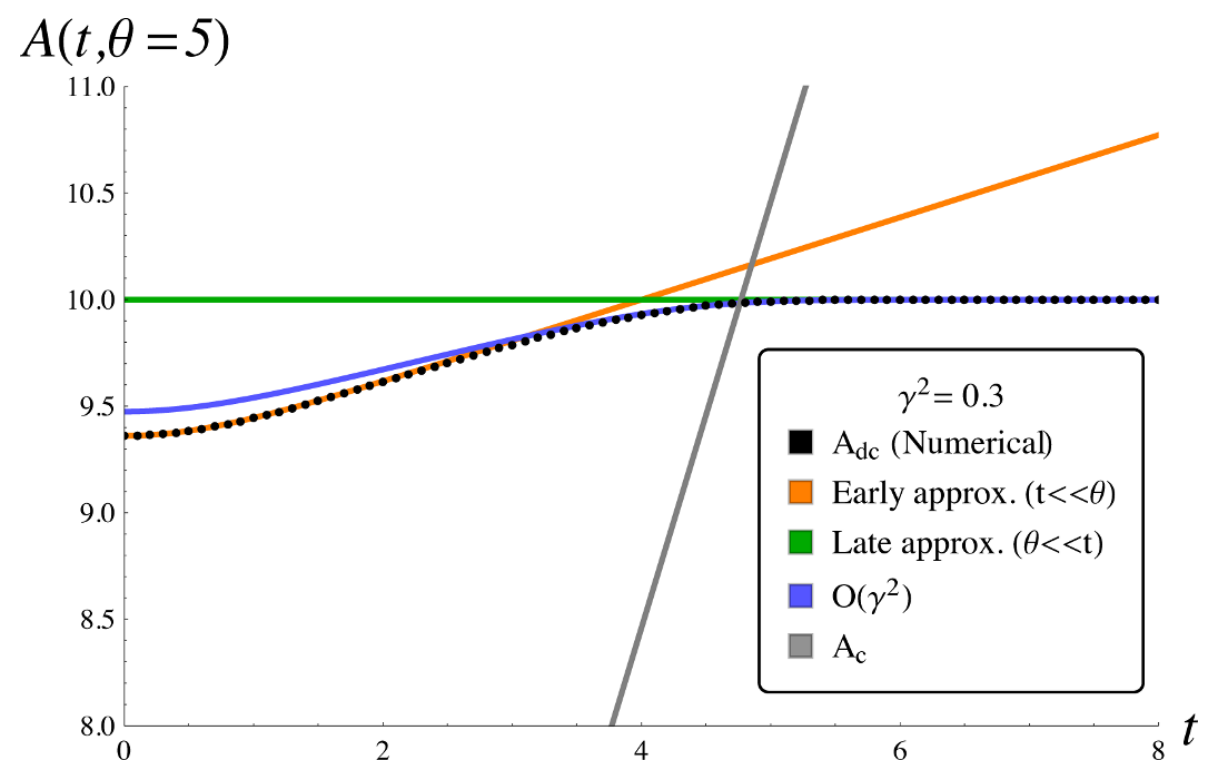

Figure 4. The time $t$ dependence of the extremal surface area $A$ for a subsystem $\theta=5$, in the disconnected phase (black dotted line, numerically obtained) and in the connected phase (gray line). The phase transition from the connected phase to the disconnected phase occurs at their intersection point $t=t_{c}$. The disconnected phase surface area $A_{d c}$ is initially well approximated by the early time limit approximation (3.22) (orange line), and finally well approximated by the late time limit approximation (3.26) (green line). The whole time-dependence of $A_{d c}$ is qualitatively reproduced by the calculation (3.27) up to $\mathcal{O}\left(\gamma^{2}\right)$ (blue line).

It is difficult to determine the critical time $t_{c}$ analytically for arbitrary $\gamma$ and $\theta_{\infty}$, because one need to evaluate the disconnected surface area (3.15) around the difficult time region $t_{\infty} \sim \theta_{\infty}$. Here we evaluate it perturbatively around $\gamma=0$ up to the second order. The detail of the calculation is given in appendix A. By equating (3.5) and (3.27), we obtain

$$
t_{c} \simeq \theta_{\infty}-2.058 \gamma^{2}+\mathcal{O}\left(\gamma^{4}\right)
$$

Note that the coefficient of $\gamma^{2}$ does not depend on the size of the subsystem $\theta_{\infty}$ or $r_{0}$.

We can also solve the equations of motion for the disconnected extremal surface numerically, to calculate the accurate time-dependence of $A_{d c}$. The result is plotted in Figure 4, together with the $\gamma^{2}$-perturbation, the early time and late time approximations discussed in the last section. The figure shows that the $\gamma^{2}$-perturbation gives quite a good approximation around $t_{\infty} \sim t_{c}$.

$\gamma^{2} \rightarrow \frac{1}{2}$. When $\gamma^{2}$ is very close to $\frac{1}{2}$, the time evolution of the entanglement entropy does not exhibit a phase transition for a large range of $\theta_{\infty}$. The minimal value $\theta_{c}$ of the subsystem size $\theta_{\infty}$ necessary for the phase transition to happen is determined by solving

$$
A_{d c}\left(t_{\infty}=0, \theta_{\infty}=\theta_{c}, \gamma^{2}\right)=A_{c}\left(t_{\infty}=0, \gamma^{2}\right)
$$




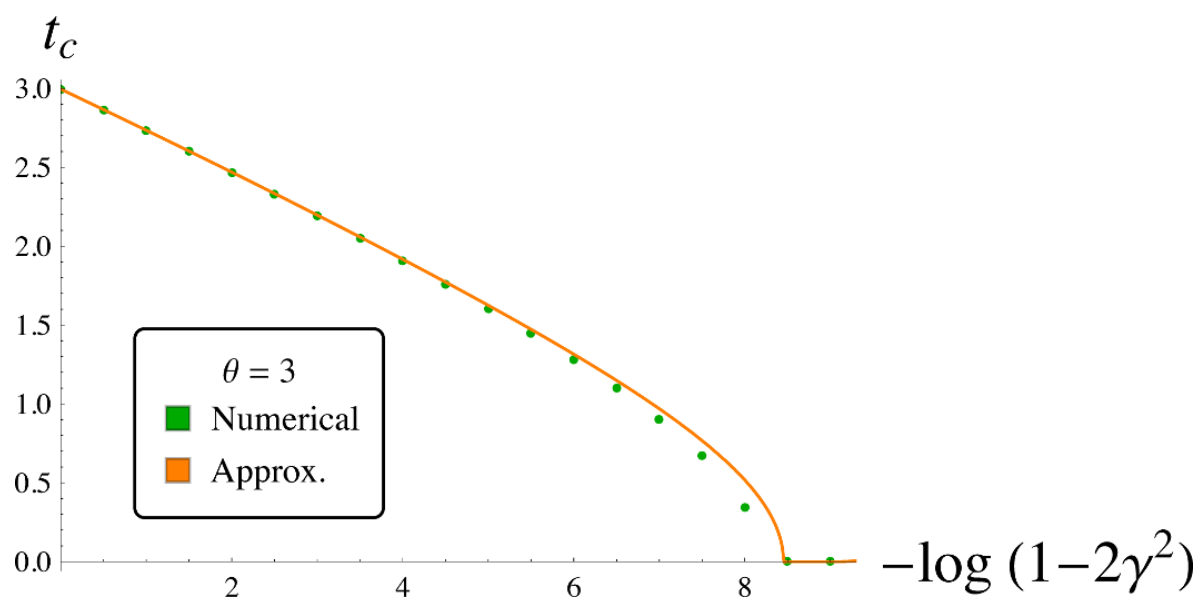

Figure 5. The $\gamma^{2}$ dependence of the transition time $t_{c}$ of a subsystem $\theta=3$. The green dots are obtained by calculating the disconnected phase surface area $A_{d c}$ numerically. The approx line (orange line) is obtained by substituting the disconnected phase surface area $A_{d c}(3.27)$ calculated up to $\mathcal{O}\left(\gamma^{2}\right)$. The transition time $t_{c}$ decreases with $-\log \left(1 / 2-\gamma^{2}\right)$ almost linearly, and the connected phase disappears with sufficiently large $\gamma^{2}$.

By using the early-time expression (3.22) for the left hand side, we can solve this equation $\mathrm{as}^{3}$

$$
\theta_{c} \simeq \frac{1}{2 \sqrt{2} r_{0}}\left(-\log \left(1-2 \gamma^{2}\right)-2 \log 2\right)
$$

When $\theta \leq \theta_{c}$, the phase corresponding to the disconnected surface is realized from the initial time $t_{\infty}=0$. Furthermore, the initial entanglement entropy is proportional to the size of the subsystem $(\propto \theta)$, which can be also seen by using the early-time approximation. This is one of the very peculiar point in the $\gamma^{2} \rightarrow \frac{1}{2}$ limit.

In summary, although the time evolution of the holographic entanglement entropy in the Janus black hole is similar to that of the BTZ black hole, there are some significant differences. First, the growth rate of the holographic entanglement entropy remains positive even after the phase transition, whereas the HEE for BTZ black hole is constant (i.e., the growth rate is zero) after $t_{c}$. Second, the introduction of the parameter $\gamma$ makes the connected surface less easy to realize. It in turn brings a result that the transition time $t_{c}$ becomes earlier. Accordingly, the "critical value" $\theta_{c}$ for the subsystem size increases as $\gamma^{2}$ grows and approaches to $\frac{1}{2}$. Third, there is a nonzero initial entanglement entropy in general. In particular, when $\gamma^{2}$ is very close to $\frac{1}{2}$, it is proportional to the size of the subsystem even for relatively large $\theta_{\infty}$ 's. These results are hard to be understood in the quasi-particle picture, in contrast to the BTZ results.

It is also interesting to see the time evolution of the mutual information defined by

$$
I(A ; B)=S(A)+S(B)-S(A \cup B)
$$

\footnotetext{
${ }^{3}$ We dropped subleading terms for $1-2 \gamma^{2}$, because in (3.22) we already used the $r_{0} \theta \gg 1$ approximation, which in turn implies $1-2 \gamma^{2} \ll 1$ here.
} 
which measures the entanglement between two subsystems $A$ and $B$. Here we take the subsystem $A$ to be an interval $-\theta_{\infty}<\theta<\theta_{\infty}$ in the right CFT, and $B$ to be the same interval in the left CFT. The original subsystem we have been considering is the union of them. Therefore the $I(A ; B)$ eventually vanishes in the disconnected phase, $t_{\infty} \geq t_{c}$. For BTZ black holes this critical time is given by half the size of the subsystem $t_{c}^{(\mathrm{BTZ})}=\theta_{\infty}$ in the high temperature limit. In [16], they considered the perturbation of BTZ black holes by a shock wave sent from one boundary, and found that the critical time becomes shorter by so called scrambling time. Here we see that our $\gamma$-deformation also leads to earlier critical times. The main difference between our case and theirs is that the deviation of the critical time from the BTZ value $t_{c}-t_{c}^{(\mathrm{BTZ})}$ is proportional to the inverse temperature $\beta$ in their case, while it is not in our case.

\section{Conclusions}

In this paper, we considered a three-dimensional, time-dependent two-sided black hole (Janus black hole) which can be regarded as a one parameter generalization of the BTZ black hole. This black hole contains a long wormhole region, which is causally disconnected from the conformal boundaries. The black hole is conjectured to be the dual of a particular CFT state (2.14). The question here is how the information on the long wormhole region is encoded in the dual CFT state.

As a first step to answer this question, we calculated the time evolution of a holographic entanglement entropy $S_{A}$ in the black hole geometry, where the subsystem $A$ is the disjoint union of a region in the original CFT and a region in the thermofield double. In the calculation of the entropy, we considered the area of two (disconnected and connected) extremal surfaces in the black hole geometry.

In BTZ black hole geometry, the connected surface is initially chosen, then after the critical time $t_{c}$ which is proportional to the size of the subsystem, the disconnected surface becomes chosen. Although the behavior in the Janus black hole geometry shares many similarities to the BTZ case, there are two notable differences. First of all, we showed that the critical time is shorter than that in the BTZ case. Intuitively, this is because the Janus black hole has a longer wormhole region, therefore the length of the connected surface becomes longer than that of the BTZ black hole. We computed this critical time up to the second order of the deformation parameter $\gamma$. Secondly, we found that the disconnected surface is always chosen, when $\gamma^{2}$ gets sufficiently close to $1 / 2$ with the subsystem size fixed, namely, when the wormhole region is sufficiently long.

In Figure 3, we numerically plotted the bulk region where the disconnected surface can arrive, and we found that outside the apparent horizon, there exists a barrier which any disconnected surface cannot go beyond. As a result, after the phase transition, the black hole interior region that the entanglement entropy can probe is rather limited. This limitation is especially strong in the above case when $\gamma^{2}$ is close to $1 / 2$.

In [14], it was shown that if we take subsystem $A$ to be the total space of the left CFT, the extremal surface which computes the holographic entanglement entropy has to be located in the causal shadow. This property is necessary for the holographic entanglement 
entropy formula to respect the CFT causality. We can easily check this condition in the Janus black hole, because in the large $\theta_{\infty}$ limit the corresponding extremal surface localizes at the origin $(y, t)=(0,0)$ (or $(\mu, \tau)=(0,0)$ in the coordinate $(2.1))$.

There are several outlooks for this work. It would be interesting to calculate the entanglement entropy on the dual CFT side. One candidate CFT is a free fermion system [30], for which the explicit form of the twist operator is known [31]. Figure 3 seems to show that it is not possible for the disconnected surface to penetrate the apparent horizon of the Janus black hole, and it would be interesting to prove this directly like [32].

\section{Acknowledgments}

The authors thank Matthew Headrick, Tatsuma Nishioka and Tadashi Takayanagi for fruitful discussions. The work of Y.N. was supported by Japan Society for the Promotion of Science (JSPS) Research Fellowship for Young Scientists, in part by JSPS Grant-inAid for JSPS Fellows, and also in part by World Premier International Research Center Initiative (WPI) from Ministry of Education, Culture, Sports, Science and Technology (MEXT) of Japan. The work of N. O. was supported by the Special Postdoctoral Researcher (SPDR) Program of RIKEN and in part by the interdisciplinary Theoretical Science (iTHES) Project of RIKEN. The work of T. U. was supported in part by the National Science Foundation under Grant No. NSF PHY-25915. The work of T. U. was supported by JSPS Postdoctoral Research Fellowship for Young Scientists and in part by JSPS Grantin-Aid for JSPS Fellows, in the earlier stage of this work.

\section{A The $\gamma$-expansion of holographic entanglement entropy}

In this section, we compute the entanglement entropy and the phase transition time in the leading order of $\gamma$-expansion.

Expansion of $\boldsymbol{y}_{*}$. First, let us expand (3.14). The integrand in the left hand side is expanded as

$$
\frac{\tilde{g}(y)^{2}}{\sqrt{\tilde{g}\left(y_{*}\right)^{2}-\tilde{g}(y)^{2}}}=\frac{\operatorname{sech}^{2} y}{\sqrt{\operatorname{sech}^{2} y_{*}-\operatorname{sech}^{2} y}}+\frac{\operatorname{sech}^{2} y\left(2-\operatorname{sech}^{2} y+\operatorname{sech}^{2} y_{*}\right)}{4 \sqrt{\operatorname{sech}^{2} y_{*}-\operatorname{sech}^{2} y}} \gamma^{2}+\mathcal{O}\left(\gamma^{4}\right),
$$

then

$$
\begin{gathered}
\int_{y_{*}}^{y_{\infty}} d y \frac{\tilde{g}(y)^{2}}{\sqrt{\tilde{g}\left(y_{*}\right)^{2}-\tilde{g}(y)^{2}}}=\left[\tanh ^{-1}\left(\frac{\cosh y_{*} \sinh y}{\sqrt{\cosh ^{2} y_{*}-\cosh ^{2} y}}\right)\right]_{y_{*}}^{y_{\infty}} \\
+\left[\frac{3 \cosh ^{2} y_{*}+1}{8 \cosh ^{2} y_{*}} \tanh ^{-1}\left(\frac{\cosh y_{*} \sinh y}{\sqrt{\cosh ^{2} y_{*}-\cosh ^{2} y}}\right)\right. \\
\left.+\frac{1}{8 \cosh y_{*}} \frac{\sqrt{\cosh ^{2} y-\cosh ^{2} y_{*}}}{\sinh y}\right]_{y_{*}}^{y_{\infty}} \gamma^{2}+\mathcal{O}\left(\gamma^{4}\right) \\
=\tanh ^{-1}\left(\operatorname{sech} y_{*}\right)+\left(\frac{3 \cosh ^{2} y_{*}+1}{8 \cosh ^{2} y_{*}} \tanh ^{-1}\left(\operatorname{sech} y_{*}\right)+\frac{1}{8 \cosh y_{*}}\right) \gamma^{2}+\mathcal{O}\left(\gamma^{4}\right) .
\end{gathered}
$$


By substituting this into (3.14), we obtain

$$
\begin{aligned}
\frac{\sinh r_{0} \theta}{\cosh r_{0} t} & =\sinh \left[\tanh ^{-1}\left(\operatorname{sech} y_{*}\right)+\left(\frac{3 \cosh ^{2} y_{*}+1}{8 \cosh ^{2} y_{*}} \tanh ^{-1}\left(\operatorname{sech} y_{*}\right)+\frac{1}{8 \cosh y_{*}}\right) \gamma^{2}+\mathcal{O}\left(\gamma^{4}\right)\right] \\
& =\frac{1}{\sinh y_{*}}+\left(\frac{3 \cosh ^{2} y_{*}+1}{8 \cosh y_{*} \sinh y_{*}} \tanh ^{-1}\left(\operatorname{sech} y_{*}\right)+\frac{1}{8 \sinh y_{*}}\right) \gamma^{2}+\mathcal{O}\left(\gamma^{4}\right),
\end{aligned}
$$

leading to

$$
\sinh y_{*}=F\left[1+\left(\frac{3 F^{2}+4}{8 \sqrt{1+F^{2}}} \operatorname{coth}^{-1}\left(\sqrt{1+F^{2}}\right)+\frac{1}{8}\right) \gamma^{2}\right]+\mathcal{O}\left(\gamma^{4}\right)
$$

where

$$
F(t, \theta)=\frac{\cosh r_{0} t}{\sinh r_{0} \theta}
$$

Disconnected surface area. On the other hand, from (3.15) and (3.17), $\gamma^{2}$ expansion gives

$$
A_{d c}^{(r e n)} / L=4 \log \frac{2 \cosh r_{0} t}{r_{0} \sinh y_{*}}+\left(2+\operatorname{sech} y_{*} \tanh ^{-1}\left(\operatorname{sech} y_{*}\right)\right) \gamma^{2}+\mathcal{O}\left(\gamma^{4}\right)
$$

By using (A.4) above, this results in

$$
A_{d c}^{(r e n)} / L=4 \log \left(\frac{2}{r_{0}} \sinh r_{0} \theta\right)-\left(\frac{3 F^{2}+2}{2 \sqrt{1+F^{2}}} \operatorname{coth}^{-1}\left(\sqrt{1+F^{2}}\right)-\frac{3}{2}\right) \gamma^{2}+\mathcal{O}\left(\gamma^{4}\right) .
$$

Phase transition. The phase transition time $t_{c}$ for a fixed value of $\theta$ can be computed by an equation

$$
A_{d c}^{(r e n)}=A_{c}^{(r e n)},
$$

with the aid of (3.6) and (A.7). This equation is solved as $t=t_{c}$, where

$$
\begin{aligned}
t_{c} & =t_{c}^{(0)}+t_{c}^{(1)} \gamma^{2}+\mathcal{O}\left(\gamma^{4}\right), \\
r_{0} t_{c}^{(0)} & =\cosh ^{-1}\left(\sinh r_{0} \theta\right) \\
r_{0} t_{c}^{(1)} & =-\left(\frac{1}{2}+\frac{5}{2 \sqrt{2}} \operatorname{coth}^{-1}(\sqrt{2})\right) \frac{\sinh r_{0} \theta}{\sqrt{\sinh ^{2} r_{0} \theta-1}} \\
& \simeq-2.058 \times \frac{\sinh r_{0} \theta}{\sqrt{\sinh ^{2} r_{0} \theta-1}} .
\end{aligned}
$$

Then, in particular, in the large $\theta$ limit $\left(\theta \gg r_{0}^{-1}\right)$, we obtain

$$
t_{c} \simeq \theta-2.058 \gamma^{2}+\mathcal{O}\left(\gamma^{4}\right) .
$$

Open Access. This article is distributed under the terms of the Creative Commons Attribution License (CC-BY 4.0), which permits any use, distribution and reproduction in any medium, provided the original author(s) and source are credited. 


\section{References}

[1] D. Bak, M. Gutperle and S. Hirano, Three dimensional Janus and time-dependent black holes, JHEP 02 (2007) 068 [hep-th/0701108] [INSPIRE].

[2] S.D. Mathur, The information paradox: a pedagogical introduction, Class. Quant. Grav. 26 (2009) 224001 [arXiv:0909.1038] [INSPIRE].

[3] A. Almheiri, D. Marolf, J. Polchinski and J. Sully, Black holes: complementarity or firewalls?, JHEP 02 (2013) 062 [arXiv: 1207.3123] [INSPIRE].

[4] K. Papadodimas and S. Raju, An infalling observer in AdS/CFT, JHEP 10 (2013) 212 [arXiv: 1211.6767] [INSPIRE].

[5] J. Maldacena and L. Susskind, Cool horizons for entangled black holes, Fortsch. Phys. 61 (2013) 781 [arXiv:1306.0533] [INSPIRE].

[6] S. Ryu and T. Takayanagi, Holographic derivation of entanglement entropy from AdS/CFT, Phys. Rev. Lett. 96 (2006) 181602 [hep-th/0603001] [INSPIRE].

[7] S. Ryu and T. Takayanagi, Aspects of holographic entanglement entropy, JHEP 08 (2006) 045 [hep-th/0605073] [INSPIRE].

[8] V.E. Hubeny, M. Rangamani and T. Takayanagi, A covariant holographic entanglement entropy proposal, JHEP 07 (2007) 062 [arXiv:0705.0016] [INSPIRE].

[9] T. Hartman and J. Maldacena, Time evolution of entanglement entropy from black hole interiors, JHEP 05 (2013) 014 [arXiv: 1303.1080] [INSPIRE].

[10] P. Calabrese and J.L. Cardy, Evolution of entanglement entropy in one-dimensional systems, J. Stat. Mech. 0504 (2005) P04010 [cond-mat/0503393] [INSPIRE].

[11] T. Ugajin, Two dimensional quantum quenches and holography, arXiv:1311.2562 [INSPIRE].

[12] P. Caputa, G. Mandal and R. Sinha, Dynamical entanglement entropy with angular momentum and $\mathrm{U}(1)$ charge, JHEP 11 (2013) 052 [arXiv: 1306.4974] [INSPIRE].

[13] N. Iizuka and N. Ogawa, On the entanglement of multiple CFTs via rotating black hole interior, arXiv:1402.4548 [INSPIRE].

[14] M. Headrick, V.E. Hubeny, A. Lawrence and M. Rangamani, Causality $\& 3$ holographic entanglement entropy, JHEP 12 (2014) 162 [arXiv:1408.6300] [INSPIRE].

[15] S. Fischetti, D. Marolf and A.C. Wall, A paucity of bulk entangling surfaces: AdS wormholes with de Sitter interiors, Class. Quant. Grav. 32 (2015) 065011 [arXiv: 1409.6754] [InSPIRE].

[16] S.H. Shenker and D. Stanford, Black holes and the butterfly effect, JHEP 03 (2014) 067 [arXiv: 1306.0622] [INSPIRE].

[17] S.H. Shenker and D. Stanford, Multiple shocks, JHEP 12 (2014) 046 [arXiv:1312.3296] [INSPIRE].

[18] S.H. Shenker and D. Stanford, Stringy effects in scrambling, JHEP 05 (2015) 132 [arXiv: 1412.6087] [INSPIRE].

[19] D.A. Roberts and D. Stanford, Two-dimensional conformal field theory and the butterfly effect, arXiv: 1412.5123 [INSPIRE].

[20] D. Bak, M. Gutperle and R.A. Janik, Janus black holes, JHEP 10 (2011) 056 [arXiv: 1109.2736] [INSPIRE]. 
[21] D. Bak, M. Gutperle and A. Karch, Time dependent black holes and thermal equilibration, JHEP 12 (2007) 034 [arXiv:0708.3691] [INSPIRE].

[22] J.M. Maldacena, Eternal black holes in Anti-de Sitter, JHEP 04 (2003) 021 [hep-th/0106112] [INSPIRE].

[23] V. Balasubramanian, P. Kraus, A.E. Lawrence and S.P. Trivedi, Holographic probes of Anti-de Sitter space-times, Phys. Rev. D 59 (1999) 104021 [hep-th/9808017] [InSPIRE].

[24] A. Strominger and C. Vafa, Microscopic origin of the Bekenstein-Hawking entropy, Phys. Lett. B 379 (1996) 99 [hep-th/9601029] [INSPIRE].

[25] C.G. Callan and J.M. Maldacena, D-brane approach to black hole quantum mechanics, Nucl. Phys. B 472 (1996) 591 [hep-th/9602043] [INSPIRE].

[26] J.M. Maldacena, The large-N limit of superconformal field theories and supergravity, Int. J. Theor. Phys. 38 (1999) 1113 [Adv. Theor. Math. Phys. 2 (1998) 231] [hep-th/9711200] [INSPIRE].

[27] A. Lewkowycz and J. Maldacena, Generalized gravitational entropy, JHEP 08 (2013) 090 [arXiv: 1304.4926] [INSPIRE].

[28] I. Affleck and A.W.W. Ludwig, Universal noninteger 'ground state degeneracy' in critical quantum systems, Phys. Rev. Lett. 67 (1991) 161 [INSPIRE].

[29] T. Azeyanagi, A. Karch, T. Takayanagi and E.G. Thompson, Holographic calculation of boundary entropy, JHEP 03 (2008) 054 [arXiv:0712.1850] [INSPIRE].

[30] I.A. Morrison and M.M. Roberts, Mutual information between thermo-field doubles and disconnected holographic boundaries, JHEP 07 (2013) 081 [arXiv:1211.2887] [INSPIRE].

[31] T. Azeyanagi, T. Nishioka and T. Takayanagi, Near extremal black hole entropy as entanglement entropy via $A d S_{2} / C F T_{1}$, Phys. Rev. D 77 (2008) 064005 [arXiv:0710.2956] [INSPIRE].

[32] N. Engelhardt and A.C. Wall, Extremal surface barriers, JHEP 03 (2014) 068 [arXiv:1312.3699] [INSPIRE]. 